\title{
Diversidade de helmintos intestinais em mamíferos silvestres e domésticos na Caatinga do Parque Nacional Serra da Capivara, Sudeste do Piauí, Brasil
}

\author{
Diversity of wild and domestic mammal's intestinal helminths from the Caatinga of the \\ Parque Nacional Serra da Capivara, Southeast of Piauí, Brazil \\ Martha Lima Brandão ${ }^{1 *}$; Marcia Chame²; José Luis Passos Cordeiro ${ }^{3}$; Sérgio Augusto de Miranda Chaves ${ }^{2}$ \\ ${ }^{1}$ Curso de Mestrado em Saúde Pública, Laboratório de Ecologia, Escola Nacional de Saúde Pública Sérgio Arouca, \\ Fundação Oswaldo Cruz - FIOCRUZ \\ ${ }^{2}$ Laboratório de Ecologia, Escola Nacional de Saúde Pública Sérgio Arouca, Fundaçáo Oswaldo Cruz - FIOCRUZ \\ ${ }^{3}$ Desenvolvimento Institucional e Gestão do Trabalho, Programa Institucional Biodiversidade e Saúde, \\ Fundaçáo Oswaldo Cruz - FIOCRUZ
}

Recebido em 26 de Junho de 2008

Aceito em 10 de Agosto de 2009

\section{Resumo}

Estudos sobre biodiversidade permitem avaliaçóes ecossistêmicas e monitoramentos de impactos e mudanças ambientais. A diversidade parasitária pode refletir o processo coevolutivo entre parasitos e hospedeiros, e mudanças ambientais que permitem a perda, ganho ou a manutenção das espécies nesse processo. $\mathrm{O}$ presente trabalho utilizou espécies/morfotipos de ovos de helmintos encontrados em fezes de sete mamíferos silvestres (os grupos Dasypodidae e grandes felinos, e as espécies: Tamandua tetradactyla, Cebus apella, Alouatta caraya, Cerdocyon thous, Pecari tajacu) e dois domésticos (Canis familiaris e Sus scrofa), que cohabitam o Parque Nacional Serra da Capivara (PNSC) e seu entorno, para analisar a diversidade dos helmintos intestinais desses mamíferos. Neste trabalho, utilizou-se achados da fauna helmintológica de mamíferos silvestres e domésticos, para avaliar um possível fluxo de helmintos entre estes dois grupos hospedeiros usando Unweighted Pair Group Method with Arithmetic Mean (UPGMA) dos hospedeiros com base na composição de suas faunas helmintológicas. Os resultados indicam que a região do PNSC, ainda mantém condições ambientais que ainda propiciam a manutenção da composição da fauna de helmintos de mamíferos silvestres, diferente da encontrada nos animais domésticos.

Palavras-chave: Helmintos, diversidade, Caatinga, Parque Nacional Serra da Capivara, mamíferos.

\begin{abstract}
Biodiversity studies allow ecosystem assessment and monitoring of environmental changes and impacts. Parasite diversity could reflect the host/ parasite coevolutionary process and the environment changes that permit the loss, gain or maintenance of species. This survey used species/morphotypes of helminths eggs found in feces from seven wild mammal species (the groups Dasypodidae and Large Cats, and Tamandua tetradactyla, Cebus apella, Alouatta caraya, Cerdocyon thous, Pecari tajacu) and from two domestic species (Canis familiaris and Sus scrofa), which occur within the Serra da Capivara National Park (PNSC) and surrounding areas in order to analise the diversity of mammal intestinal helminths. This work used the helminthological fauna findings of wild and domestic mammals, to consider a possible helminth flux between these two host groups using Unweighted Pair Group Method with Arithmetic Mean (UPGMA) of the hosts based on helminthological fauna composition. The results indicate that the region of the PNSC still maintains environmental conditions that still keep wild mammal helminthological fauna composition different from the one found for domestic mammals.
\end{abstract}

Keywords: Helminths, helminths egg, Caatinga, Parque Nacional Serra da Capivara, mammals.

\footnotetext{
*Autor para correspondência: Martha Lima Brandão

Curso de Mestrado em Saúde Pública, Laboratório de Ecologia,

Escola Nacional de Saúde Pública Sérgio Arouca, Fundação Oswaldo Cruz,

Rua Leopoldo Bulhóes, 1480 Térreo - Manguinhos, Rio de Janeiro - RJ, Brasil

e-mail: martha@formaeacao.com.br

Apoio: Financiado pelo CNPq e pelo Programa de Desenvolvimento Tecnológico

em Saúde Pública/Água (PDTSP/Água - FIOCRUZ), 2004 à 2006
} 


\section{Introdução}

A análise da diversidade de parasitos em animais silvestres pode ser considerada um bom indicador de saúde dos ecossistemas, pois reflete a filogenia e as condiçôes que permitem a simbiose entre parasitos e hospedeiros e, portanto, as pressóes evolutivas sobre ambos (LYMBERY, 2005). Também possibilita a observação de fluxos migratórios e de dispersão, elos de cadeias tróficas e alteraçóes de dieta, hábitos e comportamentos de hospedeiros, além da complexidade da estrutura dos ecossistemas e guildas (BONGERS; FERRIS, 1999).

Ainda não são abundantes os trabalhos sobre diversidade de parasitos voltados para a avaliação ecossistêmica. A maioria direciona-se ao emprego de índices de diversidade para estudos filogenéticos, ou para o teste da sensibilidade e resposta desses diferentes índices de diversidade.

Faltam ainda estudos mais detalhados, principalmente inventários das faunas parasitológicas de espécies silvestres e domésticas, e avaliaçóes de suas diferenças e similaridades. Ao longo do tempo, o acúmulo dessas informaçóes possibilitará a validação do uso de helmintos para o monitoramento da saúde dos ecossistemas diante das alteraçôes ambientais observadas e mensuradas (BONGERS; FERRIS, 1999).

A identificação de ovos de helmintos em fezes é feita rotineiramente na parasitologia veterinária e em zoológicos de todo o mundo, que a utiliza como forma de diagnóstico rápido para o tratamento de parasitoses de animais de estimação e cativeiro. Porém a identificação de ovos ainda é um desafio, pois alguns helmintos produzem ovos muito semelhantes, sendo difícil a identificação da espécie, utilizando-se apenas a morfometria (SLOSS et al., 1999).

É importante para o diagnóstico mais acurado a variação (amplitude) do comprimento e largura dos ovos, com e sem projeçôes polares (quando for o caso), o número de ovos medidos, a análise da dispersão das medidas dos ovos nas populaçóes estudadas e imagens. A falta dessas informaçóes torna pouco segura a comparação do tamanho dos ovos com os dados descritos na literatura.

O objetivo deste trabalho foi documentar, por meio de formas imaturas de ovos de helmintos encontradas nas fezes, a diversidade de helmintos intestinais de mamíferos silvestres do Parque Nacional Serra da Capivara e dos domésticos de seu entorno. O intuito foi também inferir sobre a possível hipótese de que a fragmentaçáo de hábitat e o adensamento de populaçóes humanas no entorno do Parque, propiciem o fluxo de parasitos entre espécies de mamíferos silvestres e domésticos.

\section{Material e Métodos}

Amostras (total de 72) de fezes foram coletadas durante quatro expediçóes ao Parque Nacional Serra da Capivara em diferentes períodos (final da seca - outubro/2004, final das chuvas - maio/2005 e início das chuvas - dezembro/2005 e dezembro/2006). As amostras de fezes de animais domésticos foram coletadas, em março de 2007, durante o período de chuvas. Foram percorridos aproximadamente $700 \mathrm{~km}$ por expedição.
O Parque Nacional Serra da Capivara está localizado no Sudeste do Estado do Piauí (08²6’ 50" S e $08^{\circ} 54^{\prime} 23^{\prime}$ "S e $08^{\circ} 36^{\prime} 34^{\prime \prime} \mathrm{W}$ e $\left.08^{\circ} 46^{\prime} 28^{\prime \prime} \mathrm{W}\right)$, no nordeste do Brasil. Compreende uma área de 130.000 ha, com clima semiárido e vegetação característica da região - a caatinga (Figura 1 ).

Foram escolhidas espécies de mamíferos cinegéticos (animais utilizados como caça) em razão da facilidade de identificação das fezes e da relação histórica da população local com o uso desses animais na alimentação. Dentre os domésticos, cão e porco (Canis familiaris e Sus scrofa) foram as espécies escolhidas para comparação com a fauna silvestre. Cães são tradicionalmente utilizados para caçar, e os porcos são criados livres ao redor das casas. Esses costumes permitem um estreito contato entre as espécies domésticas e silvestres, facilitando a disseminação de parasitos entre esses grupos hospedeiros, característica que é um dos objetos deste estudo.

As coletas de fezes de mamíferos silvestres e domésticos cobriram a área do Parque e o seu entorno, que corresponde a um cinturão de $10 \mathrm{~km}$ de largura ao longo dos $240 \mathrm{~km}$ de perímetro da Unidade de Conservação (BRASIL, 2000), onde se estabelecem as comunidades humanas de impacto imediato sobre o Parque e vice-versa.

A identificação das fezes foi realizada de acordo com a morfometria dos propágulos fecais e da análise da dieta, que permitem a fácil diferenciaçáo entre as espécies de Primatas, Myrmecophagidae, Tayassuidae, Canidae e Felidae. Porém, essa técnica não permite a diferenciação entre as fezes das espécies de grandes felinos existentes na região e entre as fezes de espécies de Dasypodidae (CHAME, 2003). As amostras de Dasypodidae puderam ser identificadas, pois foram coletadas pela oportunidade de necropsias de animais provenientes de apreensão do Instituto Brasileiro do Meio Ambiente e dos Recursos Naturais Renováveis (IBAMA) e do encontro do animal vivo (Tolypeutes tricinctus) que, por contenção mecânica, defecou espontaneamente.

No laboratório, as amostras de fezes foram reidratadas em solução aquosa de fosfato trissódico a 0,5\% (CALLEN; CAMERON, 1960), por 72 horas, conservadas em formol acético (Solução de Railliet-Henry) e sedimentadas para preparação de lâminas, segundo

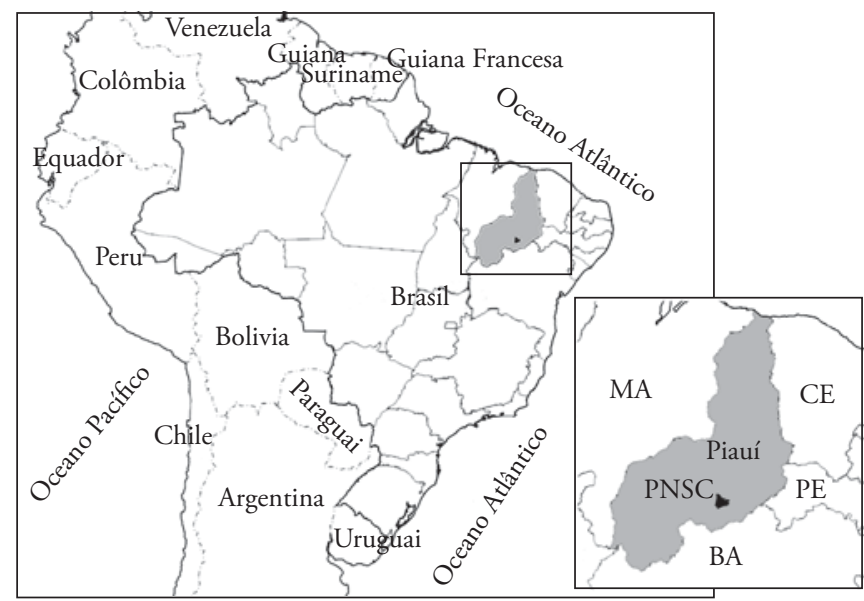

Figura 1. Localizaçáo do Parque Nacional Serra da Capivara - PI, Brasil. 
a técnica de sedimentação espontânea de Lutz (1919), revisada e adaptada por Reinhard et al. (1988). Foram confeccionadas 10 lâminas $(20 \mu \mathrm{L})$ com o sedimento de cada amostra para a análise microscópica com identificação e registro morfométrico dos ovos (comprimento e largura em micrômetros $-\mu \mathrm{m}$ ). Todos os morfotipos de ovos encontrados foram medidos e fotografados em aumento de 400 vezes.

Foram utilizadas todas as estruturas que pudessem identificar ou diferenciar um ovo, em seu menor táxon possível, tais como características e ornamentos da casca, formação embrionária e larvar, existência de opérculos e acúleos. Buscando identificar padróes métricos para as espécies ou grupos delas, foram calculados a média, o desvio padráo e a amplitude de variação do comprimento e da largura dos ovos encontrados.

A identificação dos ovos foi feita pela comparação da morfometria encontrada com a de espécies previamente descritas na literatura, para cada espécie de hospedeiro. Foram descritos como morfotipos os ovos cujas espécies não puderam ser identificadas, mas puderam ser caracterizados quanto a táxons superiores.

Para avaliar a suficiência amostral para cálculo de diversidade de parasitos, foram produzidas curvas de acumulação de espécies, nas quais a riqueza de espécies/morfotipos de helmintos foi acumulada com o aumento do número amostral de fezes (máximo de oito para todas as espécies) de cada hospedeiro analisado. A inflexão da curva aponta o tamanho amostral no qual a diversidade se estabiliza e norteia, assim, o esforço amostral empreendido para análises de biodiversidade.

Para as estimativas de riqueza e diversidade de helmintos encontrados nas espécies de hospedeiros estudadas, utilizou-se a abundância relativa de espécies/morfotipos para o conjunto de amostras $(\mathrm{N}=8)$ por espécie ou grupo de hospedeiro. Foram calculados os índices de Shannon e Simpson (KREBS, 1999) com o auxílio do software PAST ${ }^{\oplus}$ (HAMMER et al., 2001). Para verificar as diferenças da diversidade de helmintos (Shannon) entre os hospedeiros foi empregado o teste t proposto por Poole (1974).

Para caracterizar os diferentes hospedeiros quanto à composição e riqueza de sua fauna helmintológica, foi realizada uma análise de agrupamento das espécies/morfotipos de helmintos, a partir do índice de similaridade de Sorensen (que utiliza presença e ausência), pelo método de ligação média, UPGMA (LEGENDRE; LEGENDRE, 1998) com auxílio do software PC-ORD ${ }^{\oplus}$ (MCCUNE; MEFFORD, 1999). O número de grupos formados foi identificado pela média dos valores da matriz de similaridade. A escolha do índice de similaridade de Sorensen (KREBS, 1999) foi baseada na característica desse índice de valorizar mais a co-ocorrência das presenças, no caso espécie/morfotipo de helminto em espécies/ grupos de hospedeiros, do que a co-ocorrência das ausências, que poderia refletir um problema de amostragem.

\section{Resultados}

Foram analisadas oito amostras de fezes de cada uma das seguintes espécies ou grupo de espécies de mamíferos: 1) Alouatta caraya Lacepede, 1799; 2) Cebus apella Linnaeus 1758; 3) Pecari tajacu Linnaeus 1758; 4) Cerdocyon thous Linnaeus 1766; 5) grandes felinos (Panthera onca Linnaeus 1758 ou Felis concolor Linnaeus
1771); 6) Tamandua tetradactyla Linnaeus 1758 e 7) Dasypodidae (Dasypus novemcinctus Linnaeus 1758, D. septemcinctus Linnaeus 1758; Euphractus sexcinctus Linnaeus 1758 e Tolypeutes tricinctus Linnaeus 1758). Da mesma forma, foram analisadas oito amostras de cães (Canis familiaris Linnaeus 1758) e oito de porcos domésticos (Sus scrofa Linnaeus 1758), criados pelas populaçóes do entorno do Parque, totalizando 72 amostras de fezes de sete espécies de hospedeiros silvestres e duas de domésticos, coletadas nas distintas fitofisionomias do Parque e seu entorno (Figura 2).

As curvas de acumulação produzidas (espécies/morfotipos de helmintos versus o aumento do número de amostras de fezes dos hospedeiros estudados) estabilizaram-se com número de oito amostras de fezes. Somente nas curvas de acumulação obtidas para $C$. thous e S. scrofa, observa-se ainda a tendência de crescimento da riqueza de parasitos com o aumento do número amostral de fezes (Figura 3).

Os ovos foram identificados de acordo com suas características morfológicas apresentadas na Tabela 1. Foram diagnosticadas sete espécies de helmintos e 29 morfotipos nos sete hospedeiros silvestres, e 11 morfotipos nos cães e porcos domésticos (Tabela 2).

Assim, dentre as 47 espécies/morfotipos de helmintos encontradas nas fezes de mamíferos silvestres e domésticos no PNSC e entorno, a maioria, $80 \%(\mathrm{n}=37)$, é de nematódeos, duas (4\%) são de cestódeos, três (6\%) de trematódeos e duas (4\%) são de acantocéfalos. Além dessas, três espécies/morfotipos (6\%) não foram identificadas sequer quanto ao filo. Essa riqueza é também bem representada em número de famílias, superfamílias e classes de nematódeos observadas nas espécies hospedeiras (Tabela 3).

Os hospedeiros Cerdocyon thous e a Família Dasypodidae, em relação aos demais hospedeiros analisados, foram os que apresentaram a maior riqueza de helmintos $(\mathrm{R}=8)$ (Tabela 4). Os hospedeiros com menor riqueza de helmintos $(\mathrm{R}=3)$ foram os grandes felinos, com 75\% $(\mathrm{n}=5)$ das amostras apenas com Oncicola sp. (Acanthocephala), 12,5\% ( $\mathrm{n}=1)$ negativas e 12,5\% ( $\mathrm{n}=1)$ com uma espécie de Ancylostomidae e outra de Spiruridae.

Grandes felinos e T. tetradactyla apresentaram valores de H' (índice de diversidade de Shannon) de helmintos significativamente menores do que aqueles obtidos nas fezes de P. tajacu, $C$. thous, Dasypodidae e o cáo doméstico. Cerdocyon thous apresentou o maior valor de diversidade ( $\mathrm{H}^{\prime}$ ) de helmintos, mostrando uma tendência próxima da significância estatística, sendo significativamente maior do que aqueles estimados para $C$. apella $(\mathrm{p}=0,09)$, grandes felinos $(\mathrm{p}=0,02)$ e $T$. tetradactyla $(\mathrm{p}=0,03)$. Com mesma riqueza $(\mathrm{R}=8)$ na fauna helmintológica, $C$. thous não apresentou diferença significativa na diversidade em relação à Dasypodidae (Tabela 5).

Quando comparados, náo houve diferença significativa entre os valores de diversidade (Shannon) entre grupos filogeneticamente próximos (como $C$. familiares e $C$. thous; $A$. caraya e $C$. apella). Porém, levando-se em consideração a relação predador - presa (grandes felinos com $P$. tajacu e Dasypodidae), houve diferença significativa (Tabela 4).

A análise de agrupamento da composição da fauna helmintológica entre os hospedeiros aponta a formação de oito grupos entre as nove espécies de hospedeiros estudadas (Figura 4). Foram agrupadas as faunas helmintológicas de C. thous e dos grandes felinos no único grupo isolado, se for considerado um corte baseado na média $(\mathrm{M}=0,005)$ dos valores da matriz de similaridade (Figura 4). 


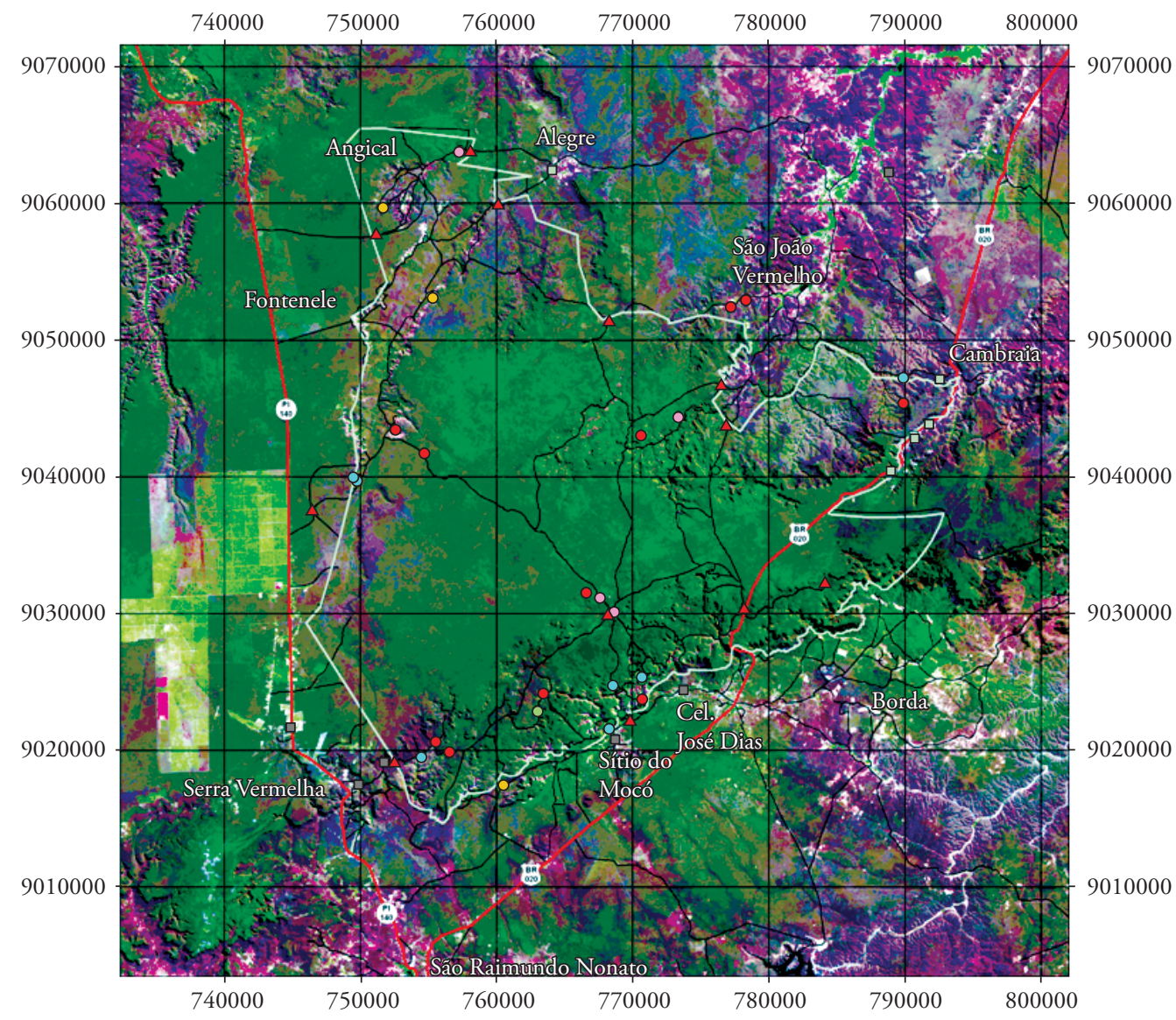

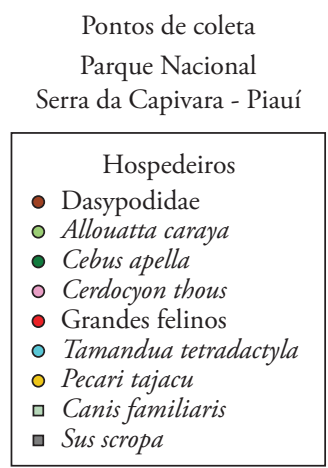
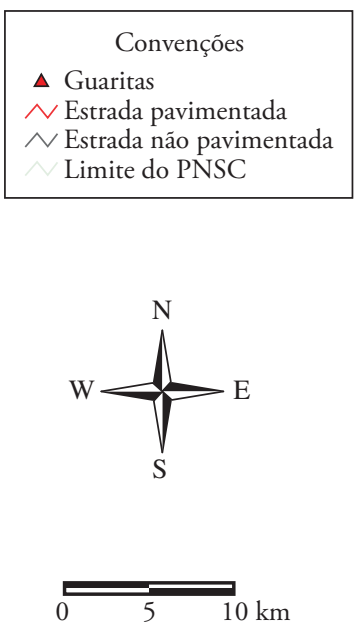

Figura 2. Pontos de coleta de fezes de animais silvestres do interior e domésticos do entorno do Parque Nacional Serra da Capivara - PI. Carta imagem elaborada à partir de composição em falsa cor (RGB543) de imagens do satélite Landsat, ano base 1998. Sistema de projeção UTM DATUM horizontal Córrego Alegre. Origem da quilometragem UTM “Equador e Meridiano 57 WGR” acrescidas as constantes: $10.000 \mathrm{~km}$ e $500 \mathrm{~km}$, respectivamente.

\section{Discussáo}

As caatingas apresentam diferenças e características únicas não existentes em nenhum outro bioma. Sendo assim, seu modelo de diversidade, provavelmente, é diferente do de outras regióes. Muitas das espécies de helmintos encontradas neste trabalho são desconhecidas da ciência ou pouco estudadas. Por isso, ainda serão necessários outros trabalhos como este na regiáo, para que se chegue a um resultado mais consistente e se aprofunde o conhecimento da complexa estrutura desse bioma.

Os dados aqui apresentados trazem uma importante contribuição para a parasitologia veterinária e também para a paleoparasitologia, já que os mamíferos estudados habitam uma das regióes mais ricas em sítios arqueológicos das Américas, com grande produção científica na área. A atual insuficiência de dados referentes à morfologia e à dimensão dos ovos de helmintos, em animais silvestres e domésticos, conduzirá a um maior aprofundamento desse tipo de abordagem multidisciplinar em ambientes como o semiárido.

Os trabalhos realizados por Chame (1988), Ferreira et al. (1989) e Araújo et al. (1989), com fezes e coprólitos de mamíferos do PNSC há cerca de 20 anos, analisaram as fezes e coprólitos de T. tetradactyla, grandes felinos, Alouatta sp., e fezes de outras espécies não analisadas neste trabalho. A presença se Giganthorhyncus echinodiscus (Acanthocephala) mantém-se na população de T. tetradactyla, no Parque, desde 625 anos d.c até os dias de hoje (ARAÚJO et al., 1989; FERREIRA et al., 1989; CHAME, 1988). Essa espécie foi encontrada somente em fezes de tamanduás, reiterando a sua especificidade a esse hospedeiro. Da mesma forma, em A. caraya, foi observada a permanência de Tripanoxyuris minutus, embora com o encontro de um único ovo entre todas as amostras analisadas. Esse oxiurídeo, de ciclo de vida direto, mantém sua forma e dimensóes tais como as descritas por Chame (1988).

Em grandes felinos, os ovos de Ancylostomatidae foram diagnosticados por Chame (1988), na região, como Ancylostoma brasiliensis. Foram encontrados também ovos dessa família em fezes de grandes felinos, porém, por causa da grande variedade entre as espécies dessa família com sobreposição de medidas, a espécie não foi diagnosticada.

A distribuição parasitária, observada nas amostras de fezes analisadas, entre indivíduos das espécies hospedeiras, está de acordo com o modelo mais comum de agregação parasitária, no qual a maior parte dos indivíduos hospedeiros de uma mesma espécie abriga poucos parasitos, enquanto poucos indivíduos abrigam muitos parasitos de uma mesma espécie ou de espécies diferentes (BEGON et al., 2006). 

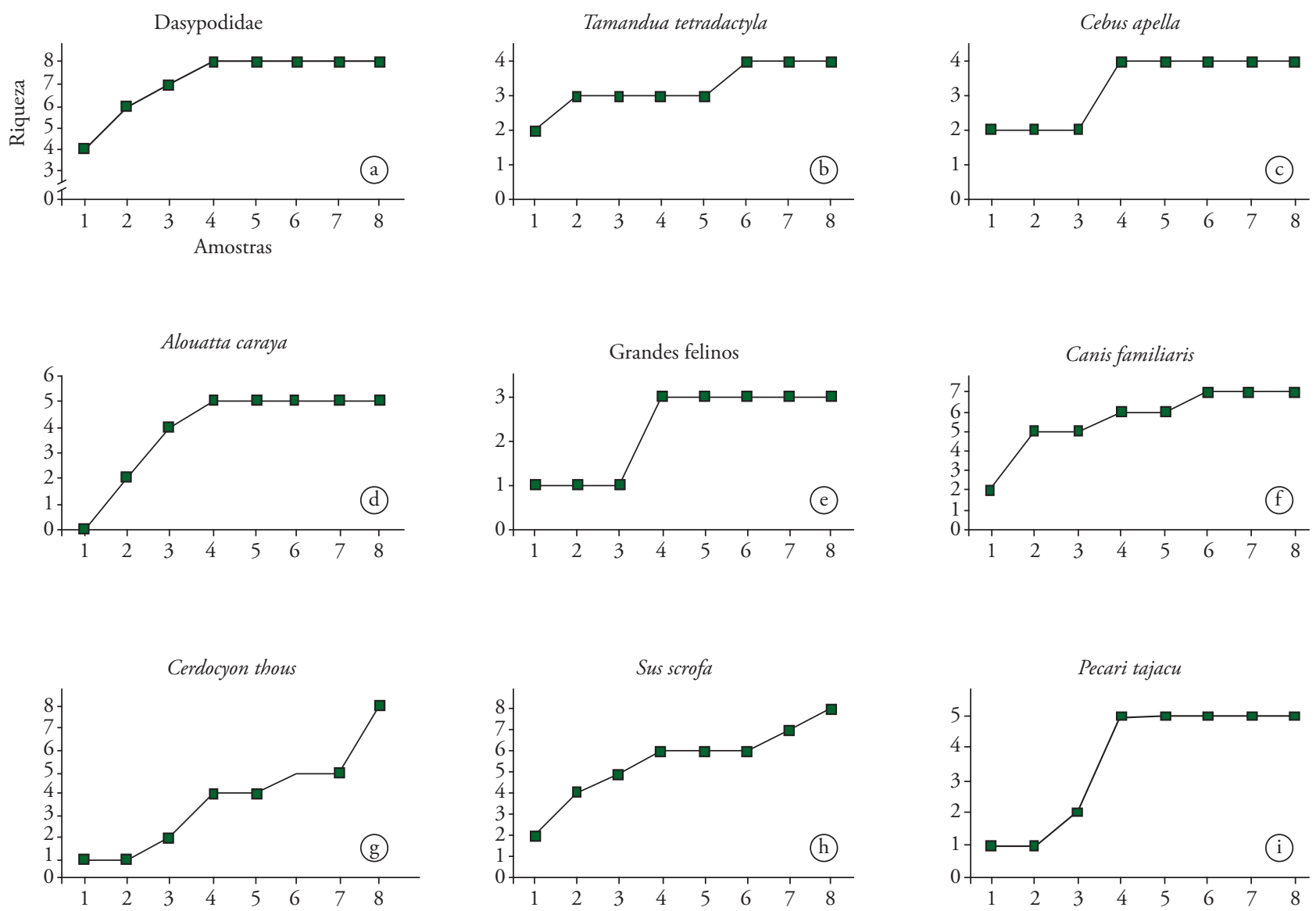

Figura 3. Curvas de acumulação de espécies/morfotipos de ovos de helmintos por número amostral de fezes de mamíferos do Parque Nacional Serra da Capivara e entorno, PI - 2004 a 2006.

Tabela 1. Descrição morfológica dos morfotipos de ovos encontrados.

\section{Táxon}

Ancylostomatidae 1

Ancylostomatidae 2

Ancylostomatidae 3

Ancylostomatidae 4

Ancylostomatidae 5

Ancylostomatidae 6

Ancylostomatidae 7

Ascarididae 1

Ascarididae 2

Ascarididae 3

Ascarididae 4

Ascarididae 5

Ascarididae 6

Ascarididae 7

Aspidoderidae 1

Aspidoderidae 2

\section{Características morfológicas}

elíptico a esférico, com casca lisa, característico da Família Ancylostomatidae. Devido à grande semelhança entre as espécies dessa família e à sua ampla distribuição em cães domésticos, não chegou-se a identificação da espécie.

oval, casca dupla, fina e lisa. Oval, com polos rombudos e arredondados, porém diferentes. Assemelha-se aos de Uncinaria sp.

elíptico, apresentando casca dupla e lisa

oval, casca dupla, fina e lisa.

elíptico, de casca espessa e extemidades bem arredondadas.

oval, casca fina e lisa contendo mórula em desenvolvimento.

elíptico, com casca lisa e polos desiguais.

casca espessa, superfície rugosa e cor castanha.

oval, casca espessa mamilonada.

elíptico, com casca mamilonada.

oval a esférico, com casca espessa dupla e irregular.

oval a esférico, com casca espessa, geralmente com três membranas visíveis.

oval a esférico com casca espessa.

casca espessa com alguns apresentando larva.

elíptico, com casca fina e irregular.

elíptico, com casca fina e lisa. Esses foram obtidos da massa fecal do trato intestinal de um indíviduo adulto necropsiado, para o qual foram também coletados os vermes adultos identificados como Aspidodera sp. 
Tabela 1. Continuação...

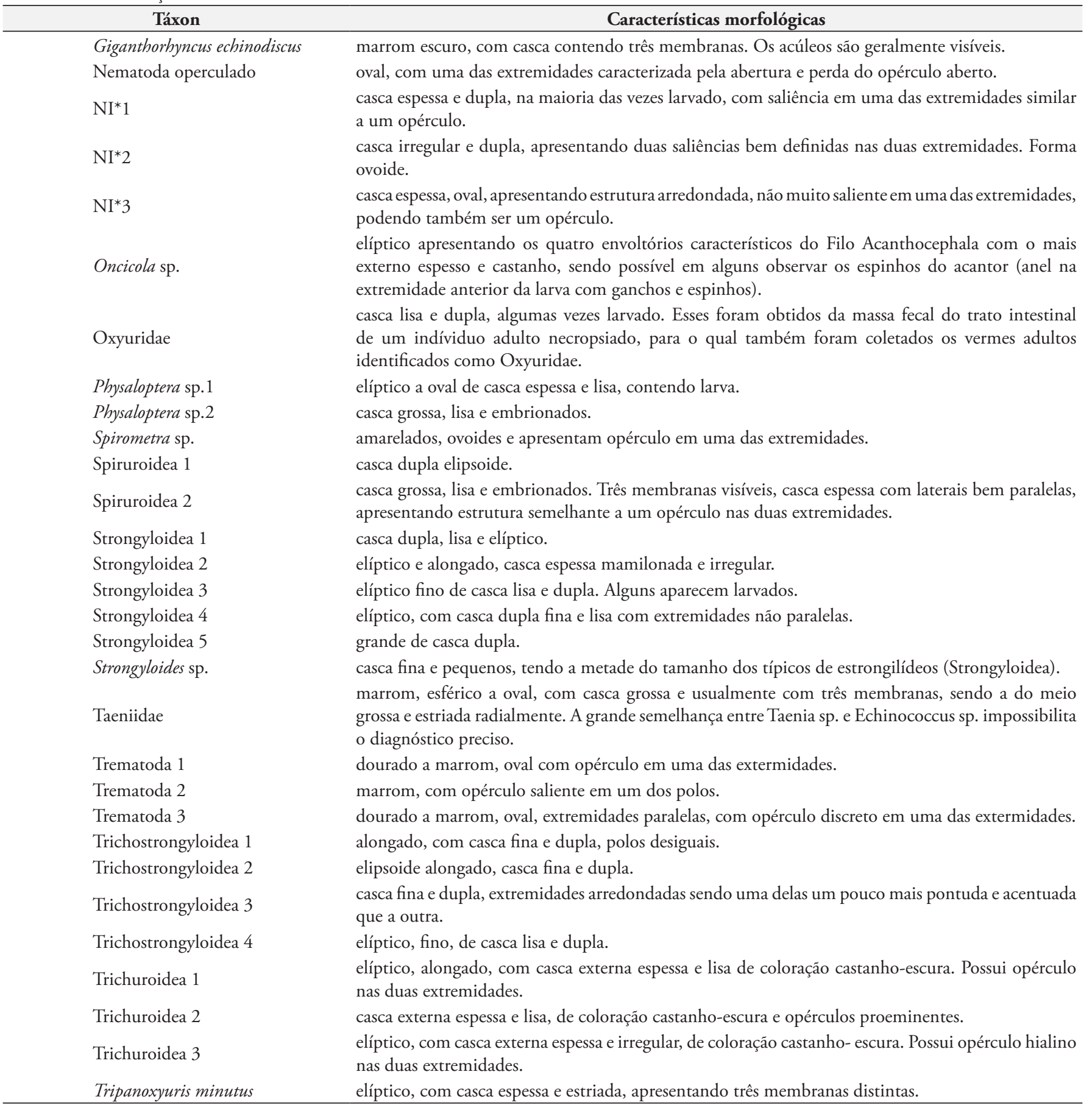

*Táxon náo identificado.

As curvas de acumulação de espécies/morfotipo de helmintos encontrados em fezes de $C$. thous e $S$. scrofa não se estabilizaram, mostrando que seriam necessárias análises de mais amostras para se conhecer a riqueza de helmintos nessas duas espécies de hospedeiros. Esse fato, provavelmente, está relacionado à grande amplitude da dieta e à característica oportunista dessas duas espécies, o que aumenta as possibilidades de novos contatos com novos parasitos. Essa observação permite inferir que as diversidades maiores de fauna helmintológica estão relacionadas às espécies de hospedeiros cujas dietas são amplas e oportunistas.
O agrupamento de $C$. thous e grandes felinos, com base na composição das suas faunas helmintológicas, em apenas um único grupo isolado entre as nove espécies de hospedeiros analisados, reforça a hipótese de que a composição de parasitos está fortemente ligada à especificidade dos helmintos com seus hospedeiros (HUGOT et al., 2001; BROOKS; MCLENAN, 1993) e também relacionada à dieta dos hospedeiros (WOOD, 2006). Reflete, portanto, os processos coevolutivos encontrados entre as ordens e famílias de hospedeiros e parasitos (BROOKS; GLEN, 1982). 
Tabela 2. Espécies / morfotipos de ovos de helmintos em fezes de mamíferos do Parque Nacional Serra da Capivara e entorno, PI - 2004 a 2006.

\begin{tabular}{|c|c|c|c|c|c|c|}
\hline \multirow[b]{2}{*}{ Hospedeiros } & \multirow[b]{2}{*}{$\begin{array}{c}\text { Helminto } \\
\text { espécie/morfotipo }\end{array}$} & \multirow[b]{2}{*}{$\begin{array}{l}\text { No ovos } \\
\text { (amostras) }\end{array}$} & \multicolumn{2}{|c|}{ Amplitude de medidas $(\mu \mathrm{m})$} & \multicolumn{2}{|c|}{ Média +/- DP } \\
\hline & & & $\begin{array}{c}\text { Comprimento } \\
\text { (s/ opérculo) }\end{array}$ & $\mathbf{L}$ & $\begin{array}{c}\text { Comprimento } \\
\text { (s/ opérculo) }\end{array}$ & $\mathbf{L}$ \\
\hline
\end{tabular}

Ordem Cingulata

Dasypodidae

Dasypus novemcinctus

Tatu verdadeiro; tatu galinha

Ascarididae 5

Aspidoderidae 1

Aspidoderidae 2

Oxyuridae

Trichostrongyloidea 3

Dasypus septemcinctus

Tatu china

Euphractus sexcinctus

Tatu peba

\section{Tolypeutes tricinctus}

Tatu bola

Ordem Pilosa

Myrmecophagidae

Tamandua tetradactyla

Tamanduá mirim;

Lapicho; Mixila

Ordem Primata

Cebidae

Cebus apella

Macaco prego;

Capuchinho

\section{Ascarididae 5}

Aspidoderidae 1

Ascarididae 5

Ancylostomatidae 6

Ascarididae 5

Aspidoderidae 1

Aspidoderidae 2

Nematoda Operculado

Trichuroidea 3

$\begin{array}{ll}7(1) & 44-54 \\ 3(1) & 50-60\end{array}$

$30-36$

$36-38$

$50,29+/-3,35$

$53,3+/-5,03$

$32,29+/-2,43$

$36,6+/-1,15$

$17(1)$

$34-56$

$24-50$

$50,47+/-5,32$

$41,06+/-5,3$

$\begin{array}{rc}60(1) & 41,6-85,8 \\ 62(2) & 40-75 \\ 3(1) & 50,6-63,8 \\ 1(1) & 52,8 \\ 11(1) & 46,8-72,8 \\ 61(1) & 70,2-85,8 \\ & (62,4-78)\end{array}$

$36,8-54,6$

$22-65$

$28,6-40$

30,8

$31,2-49,4$

$41,6-49,4$

$67,9+/-8,74$

$61,82+/-7,77$

$55,47+/-7,25$

$45,59+/-5,93$

$45,36+/-7,05$

$34,6+/-5,72$

$56,54+/-8,01$

$39,12+/-6,43$

$80,04+/-3,33$

$(71,14+/-2,89)$

$45,3+/-1,6$

Ancylostomatidae 4
Giganthorhyncus
echinodiscus
Strongyloidea 4
Trichuroidea 1

$1(1)$

$43(7)$

$14(2)$
$5(1)$

75,4

$44-65$

$20-50$

$78-78,3$

$(67,5-70,2)$
52,00

$26,4-46,8$

$55,04+/-4,49$

$34,42+/-3,59$

$8-22,5$
$37,8-40,5$

$32,00+/-9,19$

$14,64+/-5,14$

$78,24+/-0,13$

$(68,85+/-1,56)$

\begin{abstract}
Physaloptera sp. 2
Spirometra sp.

Trematoda 1

Trichuroidea 2
\end{abstract}

$\begin{array}{lc}4(1) & 45-50 \\ 8(3) & 85-119,6 \\ 1(1) & 70,00 \\ 6(3) & 67,5-85 \\ & (62,4-70,2)\end{array}$

$27,5-30$

$57,5-65$

37,5

$35-67,5$

$47,5+/-2,04$

$28,13+/-1,25$

$108,56+/-11,66$

$61,61+/-2,69$

$(62,4-70,2)$
$80,00+/-2,5$

$(68,7+/-1,25)$

Atelidae

Alouatta caraya 
Tabela 2. Continuação...

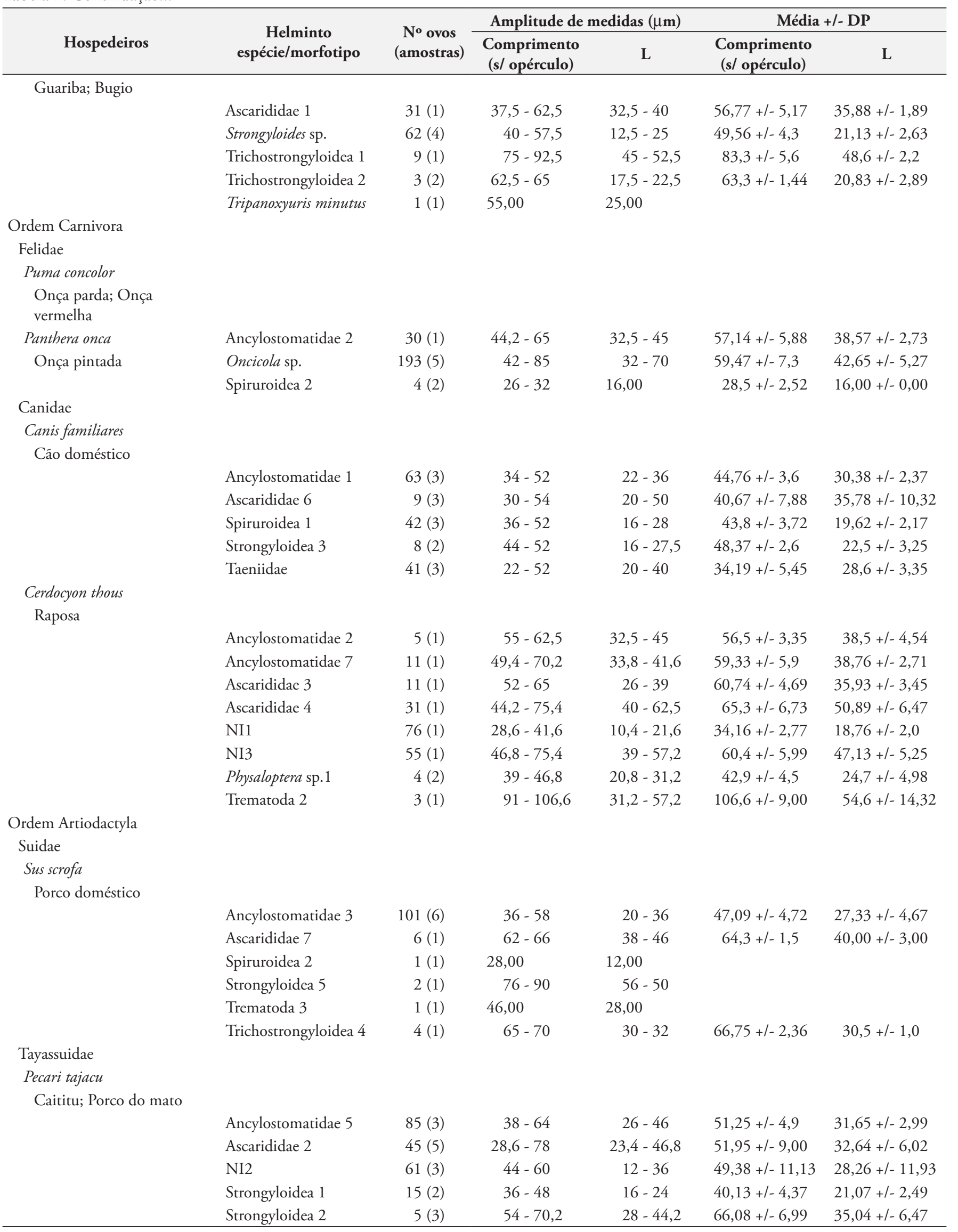

NI - Não Identificado. 
Tabela 3. Riqueza de táxons (Classe, Superfamília e Família) de helmintos intestinais identificados em amostras de fezes de mamíferos do Parque Nacional Serra da Capivara e entorno, PI - 2004 a 2007.

\begin{tabular}{|c|c|c|c|c|c|}
\hline \multirow{2}{*}{ Espécie hospedeira } & \multirow{2}{*}{ Nematoda } & \multicolumn{2}{|c|}{ Platyhelmintes } & \multirow[b]{2}{*}{ Acantocephala } & \multirow[b]{2}{*}{ NI* } \\
\hline & & Cestoda & Trematoda & & \\
\hline Dasypodidae & 8 & 0 & 0 & 0 & 0 \\
\hline Dasypus novemcinctus & 5 & 0 & 0 & 0 & 0 \\
\hline Dasypus septemcinctus & 2 & 0 & 0 & 0 & 0 \\
\hline Euphractus sexcinctus & 1 & 0 & 0 & 0 & 0 \\
\hline Tolypeutes tricinctus & 6 & 0 & 0 & 0 & 0 \\
\hline Tamandua tetradactyla & 3 & 0 & 0 & 1 & 0 \\
\hline Cebus apella & 2 & 1 & 1 & 0 & 0 \\
\hline Alouatta caraya & 5 & 0 & 0 & 0 & 0 \\
\hline Grandes Felinos & 2 & 0 & 0 & 1 & 0 \\
\hline Cerdocyon thous & 5 & 0 & 1 & 0 & 2 \\
\hline Pecari tajacu & 4 & 0 & 0 & 0 & 1 \\
\hline Canis familiaris & 4 & 1 & 0 & 0 & 0 \\
\hline Sus scrofa & 5 & 0 & 1 & 0 & 0 \\
\hline$\%$ & $80 \%$ & $4 \%$ & $6 \%$ & $4 \%$ & $6 \%$ \\
\hline
\end{tabular}

NI* - Não Identificado.

Tabela 4. Riqueza e diversidade (índices de Shannon e Simpson) de espécies/morfotipos de ovos de helmintos em mamíferos no Parque Nacional Serra da Capivara, 2004 a 2007.

\begin{tabular}{cccc}
\hline Hospedeiro & Riqueza (R) & Shannon $\left(\mathbf{H}^{\prime}\right)$ & Simpson \\
\hline Alouatta caraya & 5 & 1,39 & 0,69 \\
Cebus apella & 4 & 1,26 & 0,69 \\
Pecari tajacu & 5 & 1,56 & 0,78 \\
Cerdocyon thous & 8 & 2,04 & 0,86 \\
Grandes felinos & 3 & 0,90 & 0,53 \\
Tamandua tetradactyla & 4 & 0,98 & 0,51 \\
Dasypodidae & 8 & 1,81 & 0,79 \\
Sus scrofa & 6 & 1,54 & 0,73 \\
Canis familiaris & 5 & 1,61 & 0,80 \\
\hline
\end{tabular}

Tabela 5. Significâncias (teste t, Poole,1974) entre as diversidades (H’) de helmintos nos mamíferos hospedeiros, analisados, do Parque Nacional Serra da Capivara. O nível de significância considerado foi de $\alpha=0,1$.

\begin{tabular}{|c|c|c|c|c|c|c|c|c|c|}
\hline & $\begin{array}{c}\text { Alouatta } \\
\text { caraya }\end{array}$ & $\begin{array}{l}\text { Cebus } \\
\text { apella }\end{array}$ & $\begin{array}{l}\text { Pecari } \\
\text { tajacu }\end{array}$ & $\begin{array}{c}\text { Cerdocyon } \\
\text { thous }\end{array}$ & $\begin{array}{c}\text { Grandes } \\
\text { felinos }\end{array}$ & $\begin{array}{c}\text { Tamandua } \\
\text { tetradactyla }\end{array}$ & Dasypodidae & $\begin{array}{c}\text { Canis } \\
\text { familiaris }\end{array}$ & $\begin{array}{c}\text { Sus } \\
\text { scrofa }\end{array}$ \\
\hline Alouatta caraya & - & & & & & & & & \\
\hline Cebus apella & 0,86 & - & & & & & & & \\
\hline Pecari tajacu & 0,35 & 0,16 & - & & & & & & \\
\hline Cerdocyon thous & 1,91 & $0,09^{*}$ & 0,43 & - & & & & & \\
\hline Grandes felinos & 0,36 & 0,39 & $0,03^{*}$ & $0,02^{*}$ & - & & & & \\
\hline Tamandua tetradactyla & 0,49 & 0,55 & $0,05^{*}$ & $0,03^{*}$ & 0,82 & - & & & \\
\hline Dasypodidae & 0,24 & 0,11 & 0,57 & 0,84 & $0,02^{*}$ & $0,04^{*}$ & - & & \\
\hline Canis familiaris & 0,31 & 0,13 & 0,84 & 0,48 & $0,02^{*}$ & $0,04^{*}$ & 0,64 & - & \\
\hline Sus scrofa & 0,66 & 0,49 & 0,64 & 0,34 & 0,15 & 0,23 & 0,43 & 0,57 & - \\
\hline
\end{tabular}
${ }^{*} \mathrm{p}<0,1$.

Admitindo-se as limitaçóes dos diagnósticos feitos a partir de formas imaturas de helmintos intestinais em fezes, e utilizando-se as espécies/morfotipos encontradas como metodologia para o diagnóstico de possíveis espécies parasitas, pode-se concluir que a região do Parque Nacional Serra da Capivara ainda mantém condições ambientais que propiciam a manutenção da composição da fauna de helmintos de mamíferos silvestres diferente da dos animais domésticos.

Comparando-se os resultados deste trabalho aos relatos de helmintos nas espécies de mamíferos do PNSC em trabalhos anteriores, observa-se que, em aproximadamente 18 anos de intervalo, houve uma diferença não desprezível na composição da fauna helmintológica de algumas espécies de hospedeiros. Essa diferença é mais acentuada em primatas, nos quais foram encontrados cinco espécies/morfotipos de helmintos (Trichostrongyloidea 1, Trichostrongyloidea 2, Strongyloides sp., Ascaridiae 1 e Tripanoxyuris minutus) contra apenas uma espécie de Oxyuridae relatada nos estudos anteriores (CHAME, 1988).

É possível que a riqueza de helmintos parasitos dos mamíferos do PNSC tenha aumentado nos últimos 18 anos, porém ainda é 


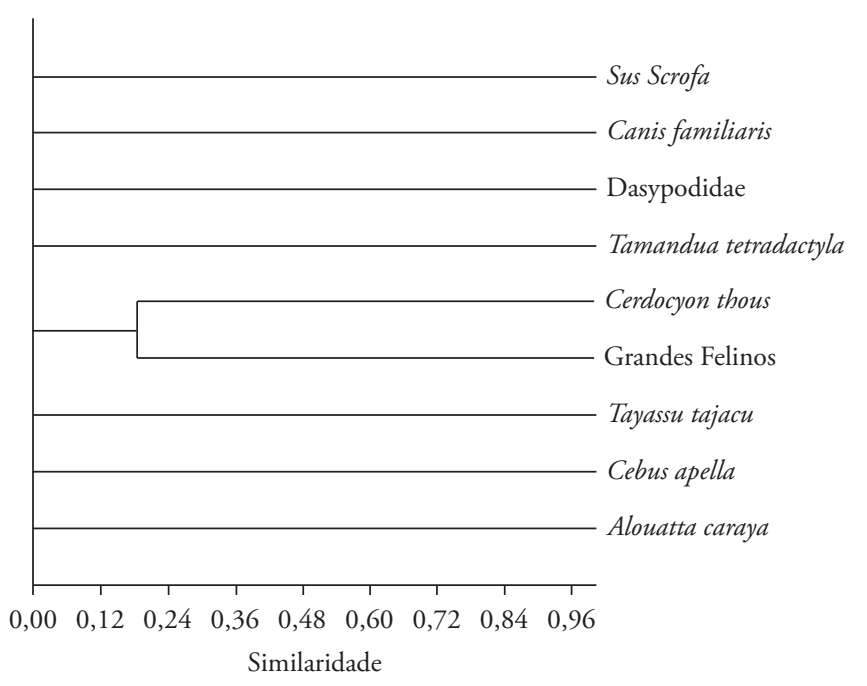

Figura 4. Dendograma da análise de agrupamento (UPGMA), usando o índice de similaridade de Sorensen, para a composição da fauna helmintológica das nove espécies de mamíferos hospedeiros do Parque Nacional Serra da Capivara, 2004 a 2007.

cedo para se fazer essa afirmação. Enquanto se observa o aumento da riqueza em algumas espécies ( $A$. caraya e Dasypodidae), observa-se também uma diminuição em outras (grandes felinos), sendo necessária a continuidade deste estudo para que seja possível o acompanhamento dessa dinâmica da pressão antrópica sobre as relaçôes parasito/ hospedeiro.

\section{Agradecimentos}

À Rita Nunes, pela confecção e ajuda prestada na leitura de algumas lâminas. Ao Dr. Jairo Barreira, pelo auxílio na confirmação do diagnóstico de helmintos dos animais domésticos. Ao Dr. José Luiz Luque, pela cuidadosa revisão e apoio.

\section{Referências}

ARAÚJO, A. et al. Strongyloides ferreirai Rodrigues, Vicente and Gomes, 1985 (Nematoda, Rhabdiasoidea) in rodent coprolites $(8,000-2,000$ years BP), from archaeological sites from Piaui, Brazil. Memórias do Instituto Oswaldo Cruz, v. 84, n. 4, p. 493-496, 1989.

BEGON, M.; TOWSEND, C. R.; HARPER, J. L. Ecology: from individuals to ecosystems. 4 ed. Oxford: Wiley Blackwell, 2006. 752 p.

BONGERS, T.; FERRIS, H. Nematode community structure as a bioindicator in environmental monitoring. Tree, v. 14, n. 6, p. 224-228, 1999.

BRASIL. Lei $\mathrm{n}^{\circ}$ 9.985, de 18 de julho de, 2000. Regulamenta o art. 225, 10, incisos I, II, III, VII da Constituição Federal institui o
Sistema Nacional de Unidades de Conservação da Natureza e dá outras providências. Diário Oficial [da] República Federativa do Brasil, Poder Executivo, Brasília, DF, 19 de julho de 2000, p. 1.

BROOKS, D. R.; GLEN, D. R. Pinworms and primates: a case study in coevolution. Proceedings of the Helminthological Society of Washington, v. 49, p. 76-85, 1982.

CALLEN, E. O.; CAMERON, T. W. M. A pre-historic diet revealed in coprolites. New Scientist, v. 8, p. 35-40, 1960.

CHAME, M. Estudo comparativo das fezes e coprólitos não humanos da região arqueológica de São Raimundo Nonato Sudeste do Piauí. Rio de Janeiro, 1988. 134 p. Dissertação (Mestrado) - Universidade Federal do Rio de Janeiro - UFRJ.

CHAME, M. Terrestrial mammal feces: a morphometric summary and description. Memórias do Instituto Oswaldo Cruz, v. 98, n. 1, p. 71-94, 2003.

FERREIRA, L. F. et al. Acantocephalan eggs in animal coprolites from archeological sites from Brazil. Memórias do Instituto Oswaldo Cruz, v. 84 , n. 2, p. 201-203, 1989.

HAMMER, Ø.; HARPER, D. A. T.; RYAN, P. D. PAST: Palaeontological Statistics software package for education and data analysis. Palaeontologia Electronica, v. 4, n. 1, p. 9, 2001.

HUGOT, J. P.; BAUJARD, P.; MORAND, S. Biodiversity in helminths and nematodes as a field of study: an overview. Nematology, v. 3, n. 3, p. 199-208, 2001.

KREBS, C. J. Ecological Methodology. 2 ed. Menlo Park: Benjamin Cummings, 1999. 620 p.

LEGENDRE, L.; LEGENDRE. P. Numerical Ecology. 2 ed. New York: Elsevier, 1998. 870 p.

LUTZ, A. O Schistosomum mansoni e a Schistosomatose segundo observações feitas no Brasil. Memórias do Instituto Oswaldo Cruz, v. 11, p. 121-155, 1919.

LYMBERY, A. J. Parasites and ecosystem health. International Journal for Parasitology, v. 35, n. 7, p. 703, 2005.

MCCUNE, B.; MEFFORD, M. J. PC-ORD: multivariate analysis of ecological data. Version 4.41. Gleneden Beach: MJM Software Desing, 1999.

POOLE, R. W. An introduction to quantitative ecology. New York: McGraw-Hill, 1974. 532 p.

REINHARD, K. J. et al. Recovery of parasite remains from coprolites and latrines: aspects of paleoparasitological technique. In: FERREIRA, L. F. Paleoparasitologia no Brasil. Rio de Janeiro: PEC/ENSP, 1988. p. $83-108$.

SLOSS, M. W.; ZAJAC, A. M.; KEMP, R. L. Parasitologia Clínica Veterinária. São Paulo: Malone Ltda., 1999. 198 p.

WOOD, M. J. Parasites entangled in food webs. Trends in Parasitology, v. 23 , n. 1, p. 8-10, 2006. 